Original article

\title{
Cancer epidemiology and trends in North Khorasan Province of Iran
}

\author{
Hosein Rafiemanesh $^{\mathrm{a}, \mathrm{b}}$, Atefe Zahedi ${ }^{\mathrm{c}}$, Mojtaba Mehtarpour ${ }^{\mathrm{d}}$, Alireza Zemestani ${ }^{\mathrm{e}}$, \\ Abbas Balouchi ${ }^{\mathrm{f}}$, Mohammad Aghaali $^{\mathrm{g}}$, Hamid Salehiniya ${ }^{\mathrm{f}, \mathrm{h}, *}$ \\ a Students research committee, School of Public Health, Shahid Beheshti University of Medical Sciences, Tehran, Iran \\ ${ }^{\mathbf{b}}$ Department of Epidemiology, School of Public Health, Shahid Beheshti University of Medical Sciences, Tehran, Iran \\ ${ }^{\mathrm{c}}$ Abadan School of Medical Sciences, Abadan, Iran \\ d Department of Health Management and Economics, School of Public Health, Tehran, Iran \\ ${ }^{\mathrm{e}}$ Road Traffic Injury Research Center, Department of Statistics and Epidemiology, Tabriz University of Medical Sciences, Tabriz, Iran \\ ${ }^{\mathrm{f}}$ Zabol University of Medical Sciences, Zabol, Iran \\ ${ }^{\mathrm{g}}$ Qom University of Medical Sciences, Qom, Iran \\ ${ }^{\mathrm{h}}$ Department of Epidemiology and Biostatistics, Tehran University of Medical Sciences, Tehran, Iran
}

\section{A R T I C L E I N F O}

\section{Keywords:}

Cancer

Epidemiology

Incidence

North Khorasan

Iran

\begin{abstract}
A B S T R A C T
Problem considered: Knowing the prevalence, incidence and cancer mortality is essential in every country for planning of control, determining priorities for prevention and treatment programs and evaluation the results to reduce the burden of cancer. Therefore, this study was performed to determine the common cancers in the north Khorasan Province.

Material and methods: The present study is a reanalysis of available data that cancer registry data in the North Khorasan province of Iran were analyzed in a five-year period (2005-2009). The reported incidence rates are standardized based on the standard population of World Health Organization and direct method. And we have presented common Cancer Trends in North Khorasan Province. Time trend analysis of incidence performed by joinpoint regression analysis was carried out using Joinpoint Regression Program.

Results: Analysis of 5-year data showed an increasing rate for the total cancer cases in both sex. During these five years, 2165 cases of cancer were registered, of these, 924 cases (42.68\%) occurred in males and 1241 (57.32\%) occurred in females. The most common cancers in North Khorasan province in five-year period were: skin (19.3\%), esophageal (14.08\%), stomach (10.62\%), breast (7.62\%) and bladder (4.57\%), respectively. For all cancers, Age standard incidence rate (ASIR) showed increasing trends in males (APC $=13.4$ ) and females $($ APC $=16.6)$, Also, there were an increasing ASIR for all of the common cancers (Except stomach and esophagus in females).

Conclusion: The results of the present study have shown that in north Khorasan the most common cancers in males were skin, esophagus and stomach; in females these were breast, skin and esophagus.
\end{abstract}

\section{Introduction}

Cancer is a major health problem around the globe, particularly in developing countries ${ }^{1,2}$ and also is the leading cause of death in the world and the third cause of death in Iran. ${ }^{3}$ It had been estimated that 12.7 million new cancer cases, 7.6 million cancer deaths would occur in 2008 in all over the world, ${ }^{3-5}$ while more than half of all deaths from cancer would occur in developing countries. ${ }^{6}$ It is predicted that the proportion of diagnosed cases will arise from about $56 \%$ in 2008 to more than $60 \%$ in 2030 in less developed countries ${ }^{4,5}$. Cancer burden in the world, especially in developing countries is increasing due to the aging and population growth. ${ }^{3,5,7}$ As well as increased acceptance of the lifestyle associated with cancer risk, such as smoking, unhealthy diet and physical inactivity. ${ }^{3,5}$ So that even if current rates remain unchanged, $50 \%$ increase in 2020 is expected which is equal to 15 million new cancer cases and 10 million new cancer death. ${ }^{7}$ It is reported that the annual cancer incidence rate in Iran is 98 to100 per $100,000 .^{3}$

Few studies have showed the association between incidence and mortality with human development index (HDI) ${ }^{8,9}$; and is changing incidence and morphology of cancers ${ }^{10,11}$ So knowing the prevalence, incidence and cancer mortality is essential in every country for planning of control and completion of health policy and determining priorities for prevention and treatment programs and evaluate the results to reduce the burden of cancer. ${ }^{3,7,12}$ Nowadays many of the deaths are

\footnotetext{
* Corresponding author at: Department of Epidemiology and Biostatistics, Tehran University of Medical Sciences, Tehran, Iran.

E-mail address: salehniya@razi.tums.ac.ir (H. Salehiniya).
} 
preventable, so that more than $30 \%$ of the cancer cases are preventable with Immunization against cancer-related infections (HPV, HBV) and a healthy life cycle. Control of risk factors will prevent about $40 \%$ of deaths from cancer and also with treatment and early detection; onethird of burden will be reduced. ${ }^{13,14}$ Thus, the establishments of national programs for prevent and control cancer is a necessary step of the Health promotion in community and also is an effective factor in reducing the incidence and burden of cancer. So, the cancer registration system is an important tool for the management and control of cancer. While only one-sixth of the world's population are covered by population-based cancer registration systems. ${ }^{5}$

Given that knowledge of the epidemiology and trend of the disease are beneficial to health planning and identify the causes of the disease. In this paper we will study about cancer incidence and trend of common cancer in the North Khorasan Province in 2005 to 2009.

\section{Methods}

\subsection{Data source}

This is analytic study, carried out based on re-analysis national registry of cancer (NCR), and Disease Control and Prevention (CDC) report of ministry of Health and Medical Education in Iran. Deputy for health of each university is responsible for health issues of the population and all health activities are managed by these deputies. All deputies for health have been included in the NCR. Registrar would apply the national registration software which was developed by CDC. The data are transmitted, by electronic file and also hard copy of 'Cancer Registry Data Collection Form'; this form is comprised of three parts: part I, regarding patient's identity characteristics and demographic information. Part II includes the most important findings of patient's clinical history. Part III includes preclinical findings. Quality control has been coordinated in five main areas by Cancer Office of CDC: (i) regarding completeness of coverage; (ii) completeness of details; (iii) accuracy of data; (iv) accuracy of reports; (v) accuracy of interpretation and (vi) repeated cases are deleted from national data. IARC software provides a way to identify inaccuracies in data coding. ${ }^{15}$ In this study all registered cases, were studied from 2005 to 2009 in the North Khorasan province in Iran. The extracted data were studied based on the number of cases and standardized incidence rate for both sexes and sex ratio. After collecting the information, trend of diseases during the study in both sexes and the distribution of the most common cancers in North Khorasan Province were shown.

\section{Statistical analysis}

We computed the age-Standardized Incidence Rate (ASIR) using direct standardized method and world standard population. To describe incidence time trends, we used Joinpoint Regression Program, Version 4.1.1.1 October $2014 .^{2}$ The analysis contained logarithmic transformation of the rates, standard error, maximum number of one joinpoints, and minimum of six years between zero joinpoints. default values were used for other parameters. The aim of the approach is to identify possible joinpoints where a significant change in the trend occurs. In present study 0 joinpiont (Full model) was a significant model. The final model selected was the most parsimonious of these, with the estimated annual percent change (APC) based on the trend within each segment. In describing trends, the terms "significant increase" or "significant decrease" signify that the slope of the trend was statistically significant $(\mathrm{P}<0.05)$. All statistical tests were two-sided.

\section{Results}

Analysis the 5-year data (2005-2009) of the Cancer Registry in North Khorasan Province showed during these five years, 2165 cases of cancer were registered, of these, 924 cases $(42.68 \%)$ occurred in males
Table 1

The Frequency and Percentage of Cancer Cases by Sex and Year in North Khorasan Province during 2005-2009.

\begin{tabular}{lccc}
\hline Year & Male & Female & \multirow{2}{*}{ Total (percent) } \\
\cline { 2 - 3 } & $\begin{array}{c}\text { Frequency } \\
\text { (percent) }\end{array}$ & $\begin{array}{c}\text { Frequency } \\
\text { (percent) }\end{array}$ & \\
\hline $\mathbf{2 0 0 5}$ & $177(42.11 \%)$ & $243(57.86 \%)$ & $420(100 \%)$ \\
$\mathbf{2 0 0 6}$ & $95(42.98 \%)$ & $126(57.02)$ & $221(100 \%)$ \\
$\mathbf{2 0 0 7}$ & $165(40.64 \%)$ & $241(59.36 \%)$ & $406(100 \%)$ \\
$\mathbf{2 0 0 8}$ & $249(45.44 \%)$ & $299(54.56 \%)$ & $548(100 \%)$ \\
$\mathbf{2 0 0 9}$ & $238(41.75 \%)$ & $332(58.25 \%)$ & $570(100 \%)$ \\
Total: & $924(42.68 \%)$ & $1241(57.32 \%)$ & $2165(100 \%)$ \\
$2005-2009$ & & & \\
\hline
\end{tabular}

and 1241 (57.35\%) in females. During the years of study, the highest number of cases was recorded in2009 with 570 cases and the lowest number was recorded in 2006 with 221 cases (Table 1).

The ASIR showed an increasing rate for the total cancer cases in both sexes. So that the age-standardized incidence rate for all cancers in males was 61.95 in 2005 and was increased to 73.25 in 2009 $(\mathrm{APC}=13.4)$. The age-standardized incidence rate for all cancers in females showed an increasing rate from 73.20 in 2005 to103.89 in 2009 (APC = 16.6). (Fig. 1 and Table 2).

In total, the most common cancers in north Khorasan province during the five-year period were as follows: skin (19.3\%), esophageal (14.08\%), stomach $(10.62 \%)$, breast $(7.62 \%)$, and bladder $(4.57 \%)$, respectively. The average ASIR within the years 2005 to 2009 revealed that the five most common cancers in females were skin $(11.73 \%)$, breast $(10.33 \%)$, esophageal $(10.18 \%)$, stomach $(5.09 \%)$, and colorectal $(3.08 \%)$. As these five cancers were accounts for $58 \%$ of cancers in females totally. And also based on the average ASIR during 2005 to 2009, the five most common cancers in males were skin $(16.96 \%)$, esophageal (11.32\%), stomach (11.18\%), bladder (5.13\%), and leukemia $(3.08 \%)$. As these five cancers were accounts for $61 \%$ of cancers in males totally.

Skin and breast cancer, the most common cancers in women in the north Khorasan province, had an increasing trend until 2008, but after it was dropped. So, the Age-Standardized Incidence Rates (ASIR) in 2005, 2008 and 2009 were 8.17, 16.47, 12.63 for skin cancer and 9.21, $20.08,11.39$ for breast cancer, respectively. And also, there was not a certain trend for esophageal cancer and always has fluctuated during the five-year period. The Joinpiont annual percent change (APC), though not significant, showed an increasing trend of common cancers for females (Except stomach and esophagus in females) (Table 2).

Fig. 2 shows the most common cancers in males in north Khorasan province. Generally, trend of cancer in males is increasing. The agestandardized incidence rate of skin cancer has increased in males from 11.42 in 2005 to 22.44 in 2009 . The age-standardized incidence rate of esophageal cancer in males has increased from 10.59 in 2005 to14.19 in 2009. Also, this rate for bladder cancer and leukemia has increased from 3.77 to 4.87 and 6.49 to 8.13 , respectively. The Joinpiont annual percent change (APC), though not significant, showed an increasing trend of all common cancers for females (Except stomach and esophagus in females) (Table 2).

The lowest reported cases of cancer in both males and females between 2005 and 2008 were Adrenal cancer (4 cases) and Parotid and salivary gland cancer ( 6 cases). The incidence rate for all cancers increased with increasing age in both sexes. So that in 2008, from the total of age-standardized incidence rate of cancers, $93 \%$ in males and $87 \%$ in females had occurred over the age of 50 years.

\section{Discussion}

It is estimated that about 50,800 new cases of cancer occur each year in Iran. Of which more than $53 \%$ are related to male with age- 


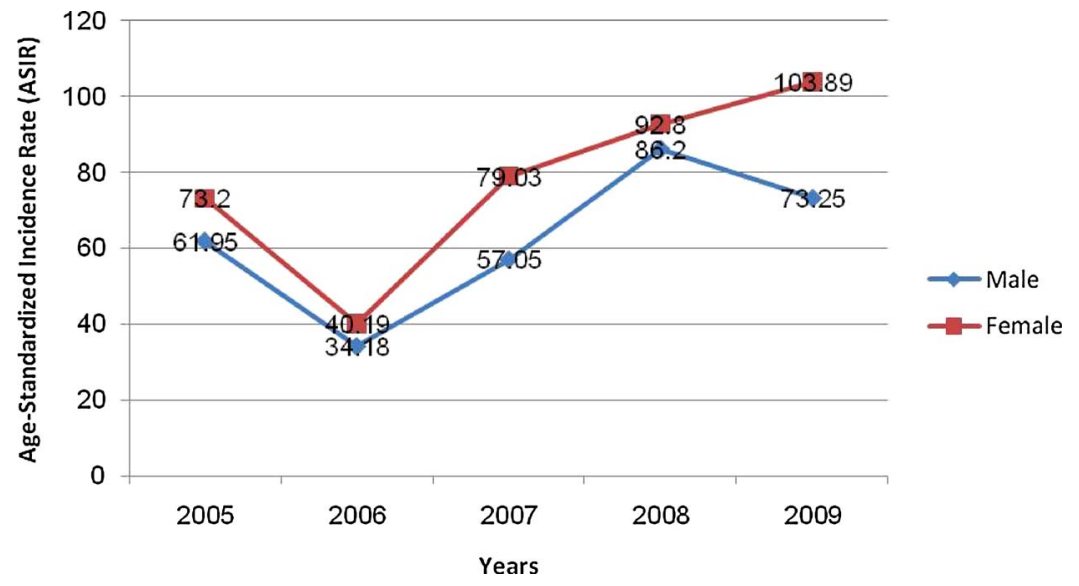

Fig. 1. ASIR trend of cancer in North Khorasan province by sex between 2005 and 2009.

standardized incidence rate of 116.8 per 100,000 in males and 93.1 per 100,000 in females. ${ }^{16}$ Although in the present study, the frequency of malignancies was more common in females than males $(57.32 \%$ vs. $42.68 \%$ ), in a study carried out by Fallah, consistent with cancer incidence data in Iran, cancer cases were more common in males (56.9\%) than females (41.9\%). ${ }^{7}$ In present study, in 2009 , the age-standardized incidence rate in males was 73.25 and 103.89 in females, respectively.

The most commonly diagnosed cancers in both sexes in Iran are: skin, breast, stomach, colorectal, bladder, prostate and leukemia. ${ }^{15}$ Result of this study showed the most common cancers in North Khorasan province in five-year period were, skin, esophageal, stomach, breast and bladder, respectively.

The most common cancer in Iranian males based on age standardized incidence rate are: Skin cancer (18.93), stomach (16.01), prostate (12.59), bladder (12.59) and colorectal (11.31). ${ }^{15}$ The Studies showed a different ranking for common cancers in different provinces of Iran. ${ }^{17-21}$ Based on the average ASIR for these 5 years, the most common cancers in males in the north Khorasan province were skin, esophageal, stomach, bladder and leukemia, respectively.

Skin cancer is the most frequent type of cancer in both sexes and on the basis of gender in the north Khorasan province. Also, skin cancer ranked first in both sexes at the country level, but according to sex, ranked second in females and first in males. In the study of Roshandel et al in Golestan province, Skin cancer wasn't among the five common cancers. ${ }^{22}$ It was the second common cancer in Esfahan, ${ }^{3}$ the fifth common cancer in Esfarayen, ${ }^{23}$ the fourth common cancer in Tehran, ${ }^{24}$ the second in East Azerbaijan ${ }^{25}$ and the most common cancer in
Markazi province. ${ }^{26}$ Differences in different geographic areas can be attributed to diversity of weather and percent of skin protection against the suns harmful rays.

Breast cancer is the most frequent cancer, ${ }^{6,27}$ and also is the leading cause of cancer death in females worldwide, which is included about $23 \%$ of all new cancer cases ${ }^{6,28}$ And $14 \%$ of all cases of death due to cancer. ${ }^{6}$ Breast cancer has decreased in some Western countries, but is increasing in many Asian and African countries. ${ }^{5}$ The Incidence rate of Breast cancer is increasing in $\operatorname{Iran}^{27,29}$ and also is the third most common cancer in both sexes which is ranks the fourth among most common cancers in the North Khorasan province. But it is in the first place with a rate of 28.25 among Iranian females. ${ }^{29,30}$ In the North Khorasan Province, breast cancer ranks second in females with the age standardized incidence rate of 10.33 , which is much lower than in the country level. In a study by fallah, Breast cancer was the most common cancer in females that was account for $19.8 \%$ of all cancer cases in females. ${ }^{7}$ While in the present study, it was accounted for $10.33 \%$ of all cancer cases in females.

Colorectal cancer is the second most common cancer in males and the second most common cancer among females in the world, with the highest incidence rate reported in Australia, New Zealand, Europe and North America, and also the lowest rate reported in Africa and southern part of Central Asia. And also is more common in males than females. ${ }^{6}$ In West Asia, the incidence rate of colorectal cancer is $13.1 .^{6}$ The colorectal cancer is the common cancer in Iranian females, so this cancer according to average incidence rate 2004-2009, is fourth most common cancer in women Sistan and Baluchestan province in $\operatorname{Iran}^{31}$ and

Table 2

Trend and annual percent change (APC) of common cancers by sex in North Khorasan Province during 2005-2009.

\begin{tabular}{|c|c|c|c|c|c|c|c|}
\hline & & 2005 & 2006 & 2007 & 2008 & 2009 & APC $(95 \% \mathrm{CI})$ \\
\hline \multirow[t]{8}{*}{ Male } & 1. Skin & 11.42 & 12.59 & 21.42 & 16.93 & 22.44 & $17.9(-1.9,41.7)$ \\
\hline & 2. Esophagus & 10.59 & 7.65 & 12.77 & 11.42 & 14.19 & $10.4(-10.1,35.5)$ \\
\hline & 3. Stomach & 12.42 & 7.65 & 10.65 & 12.62 & 12.58 & $5.4(-16.3,32.7)$ \\
\hline & 4. Hemato. Sys. & 6.49 & 0.3 & 1.24 & 5.13 & 8.13 & $39.0(-69.2,526.8)$ \\
\hline & 5. Colon \& rectum & 3.55 & 1.8 & 3.68 & 3.75 & 5.63 & $18.0(-18.3,70.5)$ \\
\hline & 6. Bladder & 3.77 & 5.0 & 5.42 & 6.59 & 4.87 & $8.2(-10.0,30.1)$ \\
\hline & 7. Prostate & 4.1 & 0.54 & 4.98 & 2.94 & 2.62 & $8.3(-60.6,197.8)$ \\
\hline & Total & 61.95 & 34.18 & 57.05 & 86.2 & 73.25 & $13.4(-18.9,58.6)$ \\
\hline \multirow[t]{8}{*}{ Female } & 1. Breast & 9.21 & 6.47 & 8.79 & 20.08 & 11.39 & $16.9(-21.4,73.8)$ \\
\hline & 2. Skin & 8.17 & 8.11 & 14.42 & 16.47 & 12.63 & $17.1(-8.4,49.7)$ \\
\hline & 3. Esophagus & 12.63 & 9.15 & 6.35 & 14.86 & 8.35 & $-3.4(-34.4,42.4)$ \\
\hline & 4. Stomach & 4.04 & 9.15 & 1.87 & 6.02 & 5.0 & $0.1(-49.4,98.0)$ \\
\hline & 5. Brain \& CNS & 1.11 & 0 & 1.57 & 2.81 & 4.13 & NA \\
\hline & 6. Thyroid & 1.98 & 0.69 & 1.45 & 2.81 & 3.17 & $26.4(-28.1,122.3)$ \\
\hline & 7. Hemato. Sys. & 3.47 & 0.38 & 0.28 & 4.02 & 3.28 & $25.2(-71.4,447.3)$ \\
\hline & Total & 73.2 & 40.19 & 79.03 & 92.8 & 103.89 & $16.6(-15.5,60.9)$ \\
\hline
\end{tabular}

NA: Not applicable. 


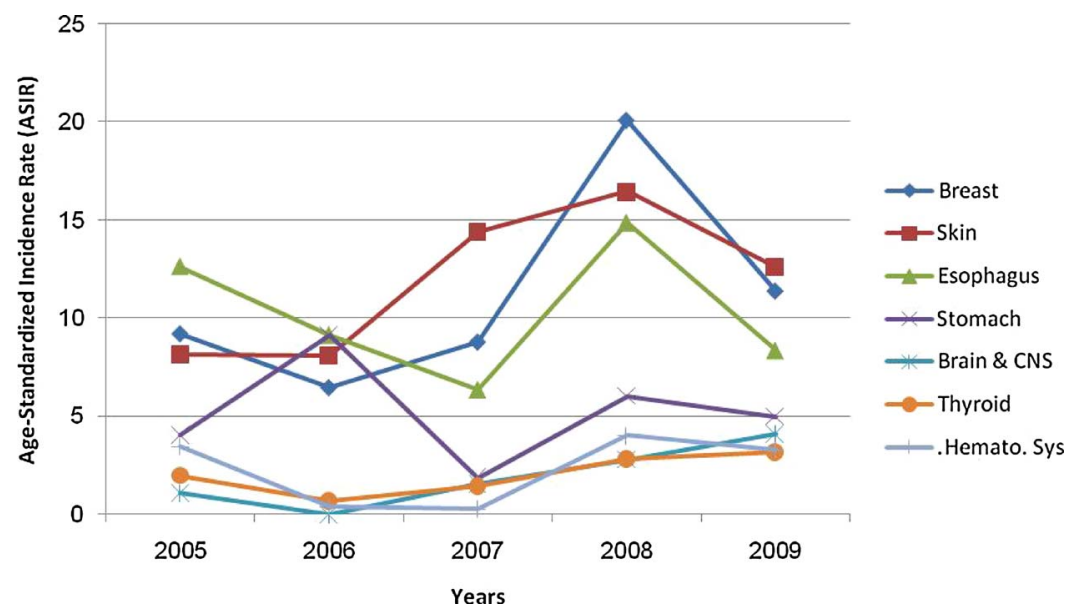

Fig. 2. Age-Standardized Incidence Rate (ASIR) trends of five cancers in North Khorasan province between 2005 and 2009.

according to sum of number cases (2004-2009) was second, fourth and fifth in Gilan, Mazandaran and Golestan province in Iran, respectively. ${ }^{21}$ In the north Khorasan province, the colorectal cancer is the fifth most common cancer in females (ASR $=3.08$ ) and is not mentioned among the five common cancers in males and these findings suggest a low incidence rate of colorectal cancer in this province. Risk factors for colorectal cancer include: smoking, physical inactivity, obesity, consumption of red and processed meat and high alcohol consumption. ${ }^{6}$ Colorectal cancer is the first common cancer in Isfahan province, $^{3}$ the second common cancer in Tehran $^{24}$ and the fifth common cancer in Markazi Province. ${ }^{26}$ Colorectal cancer was the third most common cancer in females in Khuzestan province (5.72 per $100,000)$ and also is the fourth most common cancer in males. ${ }^{32}$

Stomach cancer is decreasing in many regions of the world due to the availability of fresh fruits and vegetables and reduced intake of salted and canned foods. Stomach cancer is the fourth common cancer in males in the world, accounting for $8 \%$ of the total cancer cases in the world which $70 \%$ of new cases occur in developing countries. Stomach cancer is twice more common in males than females. Eastern Asia, Western Europe and South of America have the highest stomach cancer rates and the lowest rates are also observed in North America and many parts of Africa. These regional differences reflect differences in dietary patterns especially in European countries and the prevalence of Helicobacter pylori infection. The age-standardized incidence rate of stomach cancer in West Asia is 12.6 in males and 6.7 in females. ${ }^{6}$ Stomach cancer is third most common cancer in the north Khorasan province in both sexes, similar to that observed at the national level. While, studies conducted in different parts of the country (Islamic republic of Iran) had different results. Stomach cancer was the first common cancer in Golestan province, ${ }^{22}$ East Azerbaijan province ${ }^{25}$ and Esfarayen city. ${ }^{23}$ Stomach cancer is the most common cancer in Iranian male and female with the average age standardized Incidence rate (2003-2008) of 15.10and 7.1, respectively. ${ }^{33}$ Stomach cancer in males (ASIR $=11.18)$ and females (ASIR $=5.09$ )in North Khorasan Province is similar to the national level. Fallah's study estimated that the stomach cancer is the second common cancer in females (ASIR $=9.33$ ) and the most common cancer in males (ASIR $=22.54){ }^{7}$ Stomach cancer had lower rate in north Khorasan province compared to the national level and Fallah's study. The incidence rate of stomach cancer in province of North Khorasan is twice more common in males than females.

Esophageal cancer is twice more common among males than females. High risk areas include the north of Iran and the north central part of China in Central Asia. The United States and several Western countries are considered low-risk areas. ${ }^{6}$ Esophageal cancer is the another most common cancer in Iranian with standardized incidence rate increased from 3.51 in 2003 to 5.58 in 2008; So this increasing trend was significant for both sex. ${ }^{34}$ In a study carried out by Fallah, estimated esophageal cancer incidence rate was 12.13 in males and 8.89 in females. $^{7}$ The highest incidence rate of esophageal cancer in Iran is in Golestan province with incidence rate of 43.3 per 100,000 in males and 36.6 per 100,000 and with prevalence rate of 44 , and also the lowest incidence rate of esophageal cancer is in Kerman with the prevalence rate of $3 .^{35}$ In the north Khorasan province, esophageal cancer is the second most common cancer in males with incidence rate of 11.32 and also is the third most common cancer in females with the incidence rate of 10.18 which is three times higher than national level. Esophageal cancer was not among the five most common cancers in Tehran province $^{24}$ and Markazi province. ${ }^{26}$ These differences in esophageal cancer incidence rates can be associated with differences of people's Food habits.

Prostate Cancer is the most common cancer in males in the world. ${ }^{6}$ During the study, $9-7 \%$ of all cancers in Iranian men had prostate cancer. In total, the six-year study, 16,071 cases of prostate cancer were recorded. ${ }^{36}$ Prostate cancer is the Fifth common cancer in center of Iran (province of Qom in Iran). ${ }^{37}$ But prostate cancer wasn't among the five common cancers in the north khorasan province. In Tehran, prostate cancer is the third common cancer ${ }^{24}$ but in other study was not mentioned among five most common cancers. ${ }^{3,22,23,26}$ Also prostate cancer were among five most common cancer in three Northern provinces (Caspian Sea) in Iran (Gilan, Mazandaran and Golestan). ${ }^{21}$

\section{Conclusion}

The results of the present study have shown that the incidence rate of cancers is increasing in north Khorasan province. However, part of these increases may be related to the improvement in cancer registries. Generally, cancer incidence rates are increasing in north Khorasan province which according to the most commonly diagnosed cancers in this province, screening methods for diagnosis at early stage and educational interferences for improving the lifestyle should be used.

\section{Funding}

None declared.

\section{References}

1. Akbari ME, Hosseini SJ, Rezaee A, Hosseini MM, Rezaee I, Sheikhvatan M. Incidence of genitourinary cancers in the Islamic Republic of Iran: a survey in 2005. Asian Pac $J$ Cancer Prev. 2008;9(4):549-552.

2. Sadjadi A, Nouraie M, Ghorbani A, Alimohammadian M, Malekzadeh R. Epidemiology of breast cancer in the Islamic Republic of Iran: first results from a population-based cancer registry. East Mediterr Health J. 2009;15(6):1426-1431.

3. Mokarian F, Ramezani MA, Heydari K, Tabatabaeian M, Tavazohi H. Epidemiology and trend of cancer in Isfahan 2005-2010. J Res Medical Sci. 2011;16(9):1228. 
4. Ferlay J, Shin HR, Bray F, Forman D, Mathers C, Parkin DM. Estimates of worldwide burden of cancer in 2008: GLOBOCAN 2008. Int J Cancer. 2010;127(12):2893-2917.

5. Jemal A, Center MM, DeSantis C, Ward EM. Global patterns of cancer incidence and mortality rates and trends. Cancer Epidemiol Biomarkers Prev. 2010;19(8):1893-1907.

6. Jemal A, Bray F, Center MM, Ferlay J, Ward E, Forman D. Global cancer statistics. CA Cancer J Clin. 2011;61(2):69-90.

7. Fallah M. Cancer Incidence in Five Provinces of Iran: Ardebil, Gilan, Mazandaran, Golestan and Kerman, 1996-2000. Tehran University of Medical Sciences, Digestive Diseases Research Center; 2007.

8. Rafiemanesh H, Mehtarpour M, Khani F. Epidemiology, incidence and mortality of lung cancer and their relationship with the development index in the world. $J$ Thoracic Dis. 2016;8(6):1094-1102.

9. Rafiemanesh H, Mohammadian-Hafshejani A, Ghoncheh M, et al. Incidence and mortality of colorectal cancer and relationships with the human development index across the world. Asian Pac J Cancer Prev: APJCP. 2015;17(5):2465-2473.

10. Koohi F, Salehiniya H, Shamlou R, et al. Leukemia in Iran: epidemiology and morphology trends. Asian Pac J Cancer Prev: APJCP. 2014;16(17):7759-7763.

11. Razi S, Rafiemanesh H, Ghoncheh M, Khani Y, Salehiniya H. Changing trends of types of skin cancer in Iran. Asian Pac J Cancer Prev. 2015;16(12):4955-4958.

12. Nemati A, Abbasi I. Cost-benefit analysis in patients with cancer (esophagus, gastric) in North Khorasan Province. Eur Online J Nat Soc Sci. 2014;2(3):1138-1146.

13. Thun MJ, DeLancey JO, Center MM, Jemal A, Ward EM. The global burden of cancer: priorities for prevention. Carcinogenesis. 2010;31(1):100-110

14. De Martel C, Ferlay J, Franceschi S, et al. Global burden of cancers attributable to infections in 2008: a review and synthetic analysis. Lancet Oncol. 2012;13(6):607-615.

15. Iranian Annual Cancer Registration Report 2005-2009. Ministry of Health and Medical Education, Health Deputy Center for Disease Control and Prevention [In Persian].

16. Alireza S, Mehdi N, Ali M. Cancer occurrence in Iran in 2002, an international perspective. Asian Pac J Cancer Prev. 2005;6(3):359.

17. Rafiemanesh H, Mehtarpoor M, Mohammadian-Hafshejani A, Salehiniya H, Enayatrad M, Khazaei S. Cancer epidemiology and trends in Sistan and Baluchestan province, Iran. Med J Islam Repub Iran. 2015;29(1):752-759.

18. Keyghobadi N, Rafiemanesh H, Mohammadian-Hafshejani A, Enayatrad M, Salehiniya H. Epidemiology and trend of cancers in the province of Kerman: southeast of Iran. Asian Pac J Cancer Prev. 2015;16(4):1409-1413.

19. Rafiemanesh H, Rajaei-Behbahani N, Khani Y, et al. Incidence trend and epidemiology of common cancers in the center of Iran. Global J Health Sci. 2015;8(3):146-155.

20. Zahedi A, Rafiemanesh H, Enayatrad M, Ghoncheh M, Salehiniya H. Incidence, trends and epidemiology of cancers in north west of Iran. Asian Pac. J. Cancer Prev. 2014;16(16):7189-7193.

21. Salehiniya H, Dashdebi SG, Rafiemanesh $\mathrm{H}$, Mohammadian-Hafshejani A, Enayatrad M. Time trend analysis of cancer incidence in caspian sea, 2004-2009: a populationbased cancer registries study (northern Iran). Caspian J Int Med. 2016;7(1):25-30.
22. Semnani S, Roshandel G, Keshtkar A, et al. Golestan population-based cancer registry. golestan research center of gastroenterology and hepatology. Golestan Univ Med Sci. 2008:84-95.

23. Talebi a lm, Emani o, Haghani r. the epidemiology of common cancers in esfarayenduring 2006 to 2010. applied research national conference in public health and sustainable development.

24. Sharghi MRM, Rahimi F, Mehrazma M, et al. Distribution of cancer incidence in different neighborhods of tehran universityof medical sciences, tehran coverage razi. J Med Sci. 2011;18(89):34-45.

25. Kosha AFM, Hakimi S, et al. Cancer epidemiology in azarbayejan sharghiprovince in 2007. Med J Tabriz Univ Med Sci. 2009;32(4):74-79.

26. Mohaghegh F, Hamta A, Shariatzade MA. The study of cancer incidence and cancer registration in Markazi province between 2001 and 2006 and comparison with national statistics, Iran. Arak Univ Med Sci J. 2008;11(2):84-93.

27. Montazeri A, Vahdaninia M, Harirchi I, et al. Breast cancer in Iran: need for greater women awareness of warning signs and effective screening methods. Asia Pac Fam Med. 2008;7(1):6.

28. Harirchi I, Kolahdoozan S, Karbakhsh M, et al. Twenty years of breast cancer in Iran: downstaging without a formal screening program. Ann Oncol. 2011;22(1):93-97.

29. Rafiemanesh H, Salehiniya H, Lotfi Z. Breast cancer in iranian woman: incidence by age group, morphology and trends. Asian Pac J Cancer Prev: APJCP. 2015;17(3):1393-1397.

30. Khazaei S, Ayubi E, Mansournia MA, Rafiemanesh H. Trend of some tuberculosis indices in Iran during 25 yr period (1990-2014). J Res Health Sci. 2016;16(3):141-146.

31. Rafiemanesh H, Mehtarpoor M, Mohammadian-Hafshejani A, Salehiniya H, Enayatrad M, Khazaei S. Cancer epidemiology and trends in Sistan and Baluchestan province, Iran. Med J Islamic Republic Iran. 2015;29:254.

32. Talaiezadeh A, Tabesh H, Sattari A, Ebrahimi S. Cancer incidence in southwest of Iran: first report from khuzestan population-based cancer registry, 2002-2009. Asian Pac J Cancer Prev. 2013;14(12):7517-7522.

33. Almasi Z, Rafiemanesh H, Salehiniya H. Epidemiology characteristics and trends of incidence and morphology of stomach cancer in Iran. Asian Pac J Cancer Prev. 2015;16(7):2757-2761

34. Rafiemanesh H, Maleki F, Mohammadian-Hafshejani A, Salemi M, Salehiniya H. The trend in histological changes and the incidence of esophagus cancer in Iran (2003-2008). Int J Prev Med. 2016;7(1):31.

35. Ghavamzadeh ARDESHIR, Moussavi A, Jahani M, Rastegarpanah M, Iravani M. Esophageal cancer in Iran. Semin Oncol. 2001;28(2):153-157.

36. Pakzad R, Rafiemanesh H, Ghoncheh M, et al. Prostate cancer in Iran: trends in incidence and morphological and epidemiological characteristics. Asian Pac J Cancer Prev. 2016;17(2):839-843.

37. Rafiemanesh H, Rajaei-Behbahani N, Khani Y, Hosseini S. Incidence trend and epidemiology of common cancers in the center of Iran. Global J Health Sci. 2016;8(3):146-155. 